\title{
Unequal Randomization
}

National Cancer Institute

\section{Source}

National Cancer Institute. Unequal Randomization. NCI Thesaurus. Code C142743.

A type of sorting to allocate subjects/patients in a clinical trial into groups at different rates. 